\title{
ESTIMAÇÃO VOLUMÉTRICA DE RESÍDUOS LENHOSOS DE MADEIRA EM UMA FLORESTA TROPICAL ATRAVÉS DA KRIGAGEM ORDINÁRIA, PARAGOMINAS, ESTADO DO PARÁ ${ }^{1}$
}

\author{
VOLUMETRIC ESTIMATION OF WOOD DEBRIS TIMBER IN A TROPICAL FOREST USING \\ ORDINARY KRIGING, PARAGOMINAS, PARÁ STATE ${ }^{1}$
}

\author{
Paulo Cerqueira dos Santos ${ }^{2}$ Antônio Cordeiro de Santana ${ }^{3}$ Joaquim Carlos Barbosa Queiroz ${ }^{4}$ \\ Paulo Luiz Contente de Barros ${ }^{5}$ Ádamo Lima de Santana ${ }^{6}$
}

\begin{abstract}
RESUMO
O objetivo deste trabalho foi testar a aplicação de modelos da geoestatística para estimar o volume de resíduos lenhosos de madeira em uma área de floresta manejada no município de Paragominas, estado do Pará. Os métodos de Warren e Olsen e da Krigagem Ordinária foram utilizados para estimar o volume dos resíduos florestais em pontos amostrados e não amostrados. Os resultados confirmam que a aplicação dos métodos foi adequada, uma vez que a estimativa para o volume dos resíduos, da área em estudo, apresentou uma pequena diferença 5,2\%, em relação ao valor real obtido.
\end{abstract}

Palavras-chave: Amazônia; manejo florestal; geoestatística.

\begin{abstract}
The objective of this paper was to test the application of geo-statistical models to estimate the volume of wood timber residues in a forest area managed in Paragominas, state of Pará. The Warren and Olsen's and Ordinary Kriging methods were used to estimate the volume of forest residues in sampled and not sampled points. The results confirm that the applied methods were appropriate, given that the estimates for the volume of wood timber residues, in the area of study, showed a small difference of $5.2 \%$, when compared to the actual value obtained.
\end{abstract}

Keywords: Amazon forest; forest management; geo-statistics.

\section{INTRODUÇÃO}

$\mathrm{Na}$ exploração florestal de povoamentos e florestas naturais, o conhecimento das características dendrométricas como volume, altura e área basal, é de fundamental importância para o manejo adequado da área (MELLO, 2004). Em geral, tanto os empreendimentos ligados à exploração de florestas plantadas quanto os ligados às florestas nativas necessitam desse conhecimento, uma vez que, quanto maior for os investimentos envolvidos na implantação e/ou exploração de madeira em áreas de manejo, maior deve ser a preocupação com o planejamento para evitar prejuízos.

1 Extraído do capítulo 4 da tese de doutorado do primeiro autor.

2 Estatístico, Dr., Professor Adjunto da Universidade Federal do Pará, Caixa Postal 479, CEP 66075-110, Belém (PA), Brasil. cerquera@ufpa.br

3 Engenheiro Agrônomo, Dr., Professor Associado da Universidade Federal Rural da Amazônia, Av. Tancredo Neves, 2501, CEP 66077-901, Belém (PA), Brasil. acsantana@superig.com.br

4 Estatístico, Dr., Professor Associado da Universidade Federal do Pará, Caixa Postal 479, CEP 66075-110, Belém (PA), Brasil. joaquimqz@hotmail.com

5 Engenheiro Florestal, Dr., Professor Associado da Universidade Federal Rural da Amazônia, Av. Tancredo Neves, 2501, CEP 66077-901, Belém (PA), Brasil. paulo.contente@ufra.edu.br

6 Bacharel em Ciência da Computação, Dr., Professor Adjunto da Universidade Federal do Pará, Caixa Postal 479, CEP 66075-110, Belém (PA), Brasil. alwkynew@gmail.com

Recebido para publicação em 8/03/2011 e aceito em 20/05/2013 
No caso específico da exploração de florestas nativas, em que se faz uma seleção prévia das espécies a serem exploradas, as características dendrométricas de maior interesse, continuam sendo, o volume e a altura comercial dos fustes. A necessidade de se alcançar o máximo do aproveitamento racional dos recursos florestais dessas áreas, principalmente na Amazônia, em função da oportunidade de mercado, necessitase determinar não só o volume dos fustes, mas também de outras partes das árvores como é o caso dos galhos e sapopemas. Sabe-se, adicionalmente, que é considerável a quantidade de biomassa que é deixada na floresta quando da exploração florestal, mesmo considerando-se uma exploração de baixo impacto ao meio ambiente.

São poucos os trabalhos que procuram mostrar o quanto de biomassa é desperdiçado em função do não aproveitamento de parte das árvores tais como fustes ocos, sapopemas e copas (galhos). $\mathrm{Na}$ realidade, por falta de mercado, o aproveitamento desses resíduos é tido como inviável economicamente por uma grande parcela de empresários que atuam na exploração florestal. A maioria dos trabalhos refere-se a florestas plantadas e, naturalmente, adotam metodologias desenvolvidas para este tipo de floresta.

No caso de florestas tropicais, essas técnicas têm limitada aplicabilidade prática, em função da anatomia dos galhos e, dessa forma, propicia o aproveitamento apenas dos fustes das árvores. Poucas empresas atuantes na exploração florestal da Amazônia possuem tecnologia para o aproveitamento do que se conhece como "madeira curta" (conhecida como resíduo oriundo das galhadas). Mesmo assim, ainda não se dispõe na região de metodologia de predição que possa indicar a priori o quanto de madeira proveniente desses resíduos pode ser aproveitado, seja na produção de artefatos de madeira, ou na forma de produção de energia (sob a forma de lenha ou carvão).

Atualmente, em função da pressão exercida por entidades nacionais e internacionais ligadas às questões ambientais, algumas iniciativas de aproveitamento racional desses recursos em processo, pois, exige-se que o carvão usado pelas empresas que fabricam o ferro gusa seja proveniente de áreas de exploração certificadas. Isto induz a uma otimização maior no aproveitamento dos recursos florestais por parte dessas empresas certificadas, visto que existe a possibilidade de aumentar seus lucros sem, no entanto, aumentar os investimentos na exploração da área.

Para a estimação do volume dos resíduos de madeira na área de estudo foram utilizados os conceitos geoestatísticos de semivariograma e de krigagem. A semivariograma se preocupa com a dependência espacial entre amostras de variáveis regionalizadas. O método da Krigagem Ordinária foi o utilizado, pois, segundo Mello (2004), Isaak e Serivastava (1989) e Santos et al. (2007), é um dos métodos de inferência espacial que permite estimar informações de pontos não amostrados, a partir das informações disponíveis para os pontos amostrados da estrutura de dependência espacial estudada.

Assim, o trabalho teve como objetivo avaliar a aplicação desses modelos para o cálculo de volumetria de resíduos florestais, provenientes da exploração de impacto reduzido, numa área de manejo sustentável, localizada no município de Paragominas, estado do Pará. A hipótese subjacente a esse objetivo é de que o método geoestatístico de múltiplos pontos pode ser aplicado satisfatoriamente na estimativa de volumes de estruturas superficiais, como é o caso do volume de madeira (resíduos e fustes) e, da mesma forma que ocorre com estruturas subsuperficiais, requer um número reduzido de pontos amostrais.

\section{MATERIAIS E MÉTODOS}

A área de estudo fica localizada na Fazenda Rio Capim, com uma área de 140.658 ha, município de Paragominas, estado do Pará, distante cerca de $320 \mathrm{~km}$ de Belém, com as seguintes coordenadas geográficas $2^{\circ} 25^{\prime}$ 'e $4^{\circ} 09^{\prime} \mathrm{S}$ e $46^{\circ} 25^{\prime}$ 'e $48^{\circ} 54^{\prime} \mathrm{W} \mathrm{Gr}$ (Figura 1). O clima predominante na região, segundo a classificação de Köppen, é do tipo "Aw" (tropical chuvoso), com temperatura média anual em torno de $27,2^{\circ} \mathrm{C}$, umidade relativa do ar de aproximadamente $81 \%$ e precipitação pluviométrica média de 1766 mm/ano (PARÁ, 2009).

Os solos predominantes na região possuem várias associações dos tipos, a saber: Latossolo Amarelo, textura muito argilosa; Latossolo Amarelo, textura argilosa e Concrecionários Lateríticos; Latossolo Amarelo, textura argilosa; Latossolo Amarelo, textura média e Areias Quartzosas. Há também a presença de solos aluviais e solos indiscriminados nas áreas de várzeas. A vegetação originária da área é representada pela floresta densa da sub-região dos altos platôs do Pará-Maranhão, pela floresta densa de Planície Aluvial e dos Ferraços. Por fim, a geologia do município é representada pela 

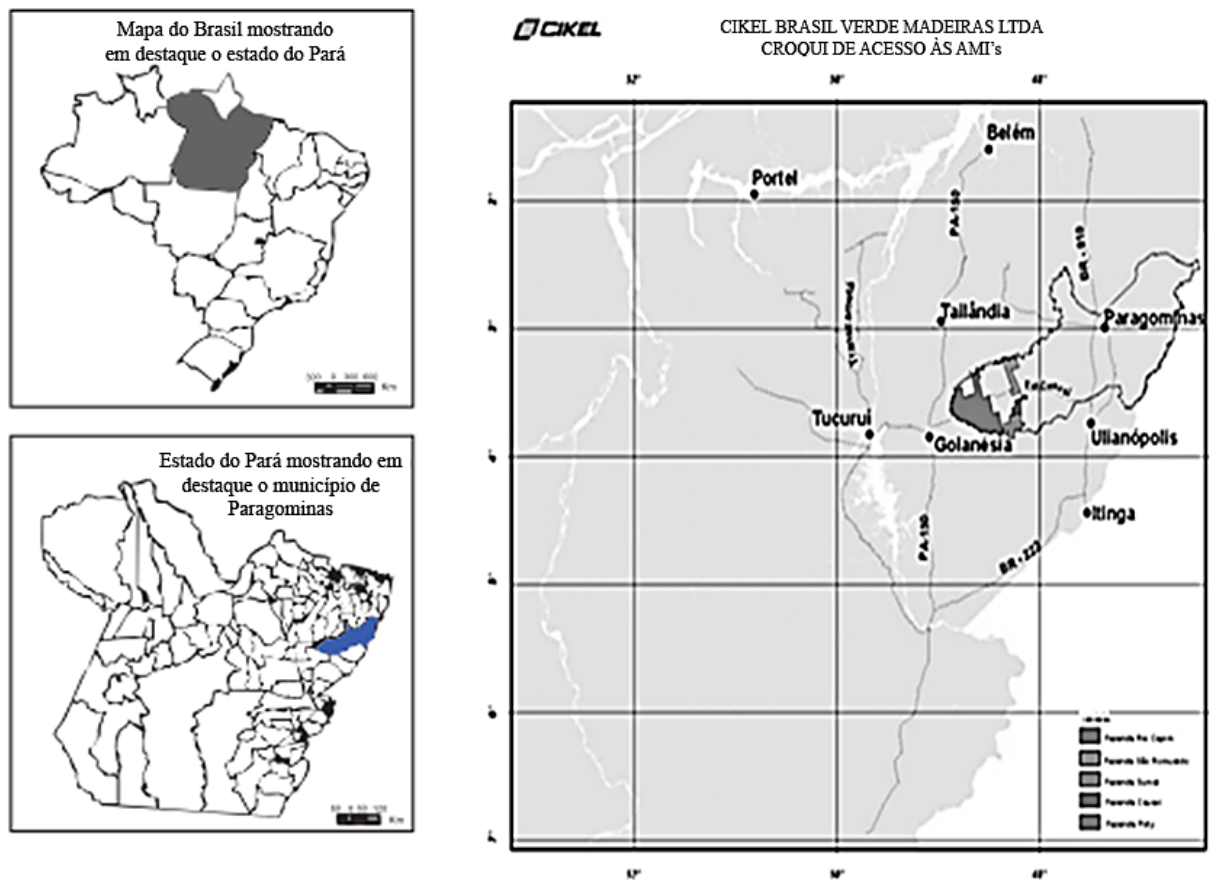

Fonte: Confederação Nacional dos Municípios - CNM/ CIKEL

FIGURA 1: Localização da área de estudo.

FIGURE 1: Location of the study area.

formação de Itapecuru, do Cretáceo, que apresenta arenitos, predominantemente vermelhos, finos, caulínicos, argilitos vermelhos laminados e calcário margoso fossilífero. Existe ainda a presença de sedimentos do Terciário, Barreiras e Quaternários subatual e recente. O relevo apresenta tabuleiros relativamente elevados e aplainados, formas colinosas dissecadas, baixos tabuleiros, terraços e várzea (PARÁ, 2009).

O levantamento das informações foi realizado numa Unidade de Trabalho (UT), com 98 ha, localizada na Unidade de Produção Anual (UPA 10), com área de 2385 ha, que se refere à parcela da área total que será explorada a cada ano.

\section{Métodos}

Neste trabalho, foi utilizada, experimentalmente, uma metodologia para a estimação volumétrica desses resíduos florestais, que se portou de forma adequada às especificidades do fenômeno em estudo. Para tanto, aplicou-se a metodologia de Warren e Olsen (1964), revisitada por Van Wagner (1968), para determinar uma estimativa a priori do volume dos resíduos florestais. Como fundamento, esta metodologia foi utilizada para determinar uma amostra aleatória dos fragmentos existentes na área de estudo e, a partir dessa amostra, determinar uma estimativa dos volumes nos pontos não amostrados. No passo seguinte, estimou-se o volume nos pontos não amostrados, por meio da Krigagem Ordinária.

Com o propósito de estimar o volume dos resíduos lenhosos provenientes da exploração florestal de impacto reduzido na área de estudo, foram utilizados os métodos de Warren e Olsen (WO) e a Krigagem Ordinária (KO). A utilização do primeiro método teve como objetivo principal obter uma amostra aleatória destes resíduos lenhosos para, posteriormente, serem utilizados como "valores amostrados" na aplicação do método da $\mathrm{KO}$, cujo objetivo é gerar uma estimativa para os volumes de resíduos nos lugares não amostrados pelo método WO (centro do quadrado de lado $50 \mathrm{~m}$ ).

$\mathrm{Na}$ KO, utilizaram-se os conceitos geoestatísticos de semivariograma e a krigagem que, conforme Landim (1998), são fundamentais para análise do comportamento de variáveis regionalizadas. Os critérios são descritos a seguir:

\section{Semivariograma}

O semivariograma mostra o grau de dependência entre amostras ao longo de um suporte específico e leva em consideração, variáveis regionalizadas, representadas por $\mathrm{Z}(\mathrm{u})$, em que u indica a localização da amostra, coletadas em 
diversos pontos, que podem estar regularmente distribuídos ou não. Para Journel e Huijbregts (1978), a função variograma, denominada $2 \gamma(h)$, é definida com a esperança matemática do quadrado da diferença entre pares de pontos separados por uma distância h:

$$
2 \gamma(h)=E\left\{[Z(x)-Z(x+h)]^{2}\right\}
$$

Como consequência, define a função semivariograma como sendo a metade da função variograma, ou seja:

$$
\hat{\gamma}(h)=\frac{1}{2 N(h)} \sum_{i=1}^{N(h)}\left[Z\left(x_{i}\right)-Z\left(x_{i}+h\right)\right]^{2}
$$

Em que $\hat{\gamma}(h)$ é a semivariância estimada entre pares de pontos, $\mathrm{N}(\mathrm{h})$ é o número de pares de pontos separados por um vetor $\mathrm{h}, \mathrm{Z}\left(\mathrm{x}_{\mathrm{i}}\right)$ é o valor da variável regionalizada no ponto $x \mathrm{e} \mathrm{Z}\left(\mathrm{x}_{\mathrm{i}}+\mathrm{h}\right)$ é o valor no ponto $x_{i}+h$. Os semivariogramas expressam o comportamento das variáveis regionalizadas, ou de seus resíduos, e mostram, dentre outras coisas, o tamanho da zona de influência em torno da amostra, a anisotropia e a continuidade.

O emprego da geoestatística, por seu turno, não impõe o pressuposto de normalidade das informações como condição necessária. Se tais informações provierem de populações normais, as inferências realizadas incorporam outras propriedades estatísticas desejáveis, tal como a Máxima Verossimilhança (MELLO, 2004). Neste sentido, foi realizada uma transformação nos dados originais pelo critério de Box-Cox, cuja expressão matemática é 1, definida da seguinte forma:

$$
Y^{\prime}=\left\{\begin{array}{l}
\log (Y), \quad \text { se } \lambda=0 \\
\frac{Y^{\lambda}-1}{\lambda}, \text { caso contrário }
\end{array}\right.
$$

Em que Y representa os valores da variável original e $\mathrm{Y}^{\prime}$ os valores transformados. Esta transformação foi realizada a fim de se corrigir os vieses da não normalidade inseridos nos dados. $\mathrm{O}$ teste utilizado para aferir a normalidade dessa variável foi o de Jarque-Bera, descrito em Santana (2003).

A ideia foi utilizar a função semivariograma para especificar o modelo experimental e, a partir desse modelo, ajustar um modelo espacial capaz de agregar toda a informação possível da estrutura de continuidade espacial. Neste trabalho, foram ajustados os modelos teóricos esférico, exponencial e gaussiano. Para cada um dos modelos ajustados, foram determinados os seguintes parâmetros: efeito pepita $\left(\mathrm{C}_{\mathrm{o}}\right)$, contribuição $\left(\mathrm{C}_{1}\right)$, alcance $(\mathrm{a})$ e patamar $\left(\mathrm{C}_{\mathrm{o}}+\mathrm{C}_{1}\right)$. De posse da estimativa destes parâmetros, foi possível determinar o grau dependência espacial (DE\%), por meio da razão entre a contribuição e o patamar. O ajustamento dos modelos foi realizado por mínimos quadrados ordinários, mínimos quadrados ponderados e máxima verossimilhança (SANTANA, 2003; GREENE, 1993).

O cálculo da Dependência Espacial (DE) é dado pela fórmula (em que $\mathrm{C}_{\mathrm{o}}$ e $\mathrm{C}_{1}$ representam, respectivamente, a variação parcial e o efeito pepita).

A avaliação do desempenho de cada um destes modelos ajustados se deu através do critério de Akaike (Akaike's Information Criterion - AIC) e foi determinado através da função lik.GRF do software 'geoR' v. 1.6-25. O AIC foi estimado com base na equação 4 (GREENE, 1993).

$$
\mathrm{AIC}=-2 \log L+2 K
$$

Em que L é a verossimilhança maximizada pelo modelo candidato e $\mathrm{K}$ o número de parâmetros deste modelo. O modelo eleito será o que apresentar um menor valor para a estatística AIC.

\section{Krigagem}

Neste estudo foi utilizada a KO por levar em consideração a variação local da média, limitada ao domínio de estacionaridade da mesma à vizinhança local $\mathrm{W}(\mathrm{u})$, centrada sobre a localização "u" a ser estimada (QUEIROZ, 2003; WANG et al., 2005). Em outras palavras, a Krigagem Ordinária utiliza médias locais estimadas a partir dos elementos amostrais vizinhos, ao invés de uma única média estacionária, como o faz um algoritmo de interpolação simples (IMAI et al., 2003; DAVIS e IERAPETRITON, 2007).

\section{Procedimentos de campo}

As estratégias estabelecidas para viabilizar os procedimentos de campo foram aplicadas na estrutura desenvolvida pela empresa exploradora, criada com base nos resultados do inventário florestal da área, que é um dos procedimentos obrigatórios da exploração florestal manejada. Assim, a área em estudo foi dividida, nos sentidos Oeste/Leste (WL) 
e Norte/Sul (NS), por linhas interceptoras de $50 \mathrm{~m}$ em $50 \mathrm{~m}$, o que contribuiu para a formação de uma malha de $50 \mathrm{~m}$ por $50 \mathrm{~m}\left(2500 \mathrm{~m}^{2}\right)$. A localização das árvores a serem retiradas foi feita através dessas coordenadas cartesianas.

A aplicação do método de Warren e Olsen (1964) foi realizada adotando-se o mesmo sentido do caminho realizado para o planejamento da exploração florestal, criado por ocasião do inventário florestal da área. Para isso, utilizou-se uma corda de $50 \mathrm{~m}$ como linha interceptora, fixando o ponto de origem em cada piquete de demarcação da área, e uma trena de $5 \mathrm{~m}$ para mensuração das circunferências dos fragmentos de madeira que foram interceptados pelo transecto (corda). Assim, foram anotadas todas as circunferências dos resíduos florestais interceptados pela corda nos sentido WL e NS.

Por outro lado, uma estimativa mais realista do volume total de resíduos da área, está diretamente relacionada ao tamanho da malha considerada, uma vez que é grande a probabilidade de que alguns fragmentos não sejam interceptados pela linha interceptadora da malha de $50 \mathrm{~m}$ x $50 \mathrm{~m}$.

Na Figura 2 (mapa de corte e arraste $n^{\circ} 04$ ), tomada como ilustração dos pontos centrais $\mathrm{P}_{1}, \mathrm{P}_{2} \mathrm{e}$ $\mathrm{P}_{3}$, visualiza-se que muitas das árvores selecionadas para corte, mesmo aquelas com queda direcional perpendicular à linha interceptora, seus galhos dificilmente seriam interceptados por esta linha e, dessa forma, contribuiriam para uma estimativa do volume total da área menor do que o valor efetivo.

Para computar os resíduos que caem fora da linha interceptora e reduzir o problema, aplicou-se o método da KO para gerar uma estimativa do volume desses fragmentos não amostrados pelo método WO, com base no volume médio de um ponto localizado no centro do quadrado de $\mathrm{L}=50 \mathrm{~m}$ (Figura 2). Portanto, o volume existente neste ponto central foi definido com base nas informações geradas a partir do método de Warren e Olsen (1964).

A aplicação do método de KO necessita que os pontos sejam identificação través de suas coordenadas retangulares ou geográficas. Este fato cria obstáculos na aplicação do método, uma vez que os resíduos lenhosos que propiciam as estimativas dos volumes a serem obtidas pela aplicação do método de $\mathrm{WO}$, a priori, não estavam correlacionadas às suas reais coordenadas, sejam retangulares ou geográficas (as coordenadas não foram identificadas por ocasião do levantamento). Conheciam-se apenas as coordenadas retangulares

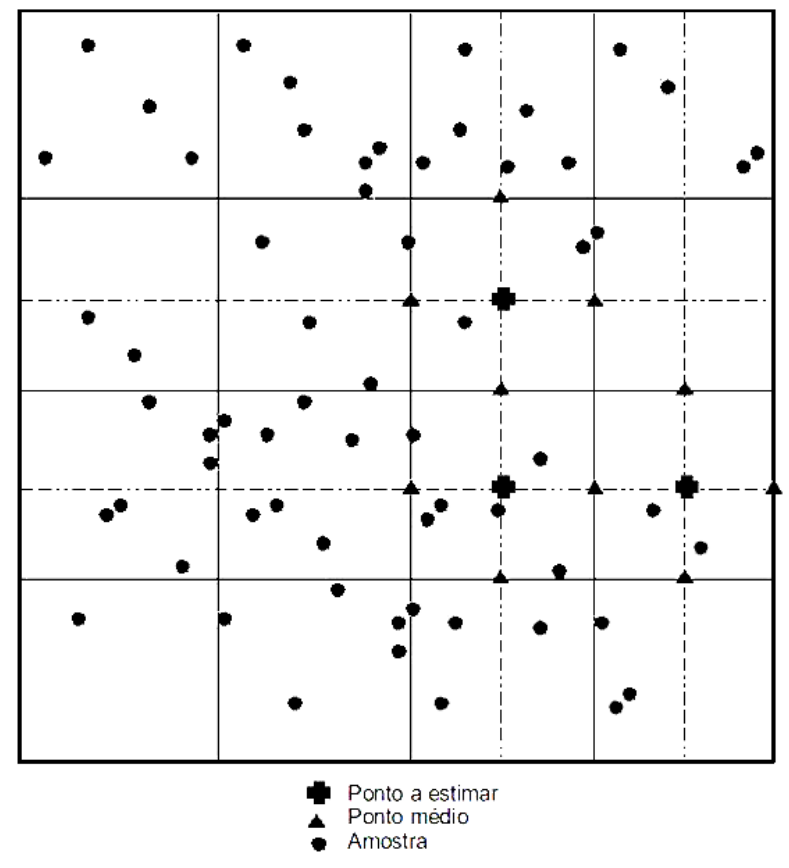

FIGURA 2: Croqui da estratégia para estimação do volume nos pontos médios.

FIGURE 2: Sketch of the strategy to estimate the volume at the midpoints.

das árvores a serem retiradas (cortadas) do povoamento florestal.

Para contornar essas questões, devemse considerar os pontos médios dos quadrados formados pelas interseções das linhas interceptoras nos sentidos WL e NS, como referência de localização dos volumes dos resíduos florestais mensurados no levantamento feito através do método de WO. Assim, o objetivo principal é estimar o volume concentrado ao redor dos pontos no centro desses quadrados: pontos $\mathrm{P}_{1}(375 ; 225)$, $\mathrm{P}_{2}(375 ; 375)$ e $\mathrm{P}_{3}(475 ; 275)$; com as informações preexistentes localizadas nos pontos médios dos lados dos mesmos.

\section{Seleção do melhor modelo ajustado}

Neste estudo foram testados os modelos teóricos esférico, exponencial e gaussiano para se avaliar qual deles melhor se ajustava aos dados de volumes obtidos através do método das linhas interceptoras para, posteriormente, ser utilizado como estimador do volume dos pontos não amostrados. 


\section{RESULTADOS E DISCUSSÕES}

\section{Análise exploratória dos dados}

O trabalho iniciou com a análise exploratória do comportamento da distribuição do volume dos resíduos, obtidos por meio do levantamento feito através das linhas interceptadoras. Os Gráficos ( $a$ e b) da Figura 3 ilustram a dispersão dos volumes com relação aos sentidos NS e WL, quando da captação dos dados. Nota-se que, nos dois casos, é possível destacar a presença de candidatos a valores discrepantes ou outliers que, segundo Diggle e Ribeiro Junior (2000), pode influenciar consideravelmente o comportamento do semivariograma, sobretudo na parte inicial do mesmo.

Neste estudo, em função das características da floresta de apresentar árvores que produzem muitos resíduos e algumas que geram um volume pequeno, decidiu-se não eliminar os valores atípicos e trabalhar com todos os valores amostrados, seguindo orientações de Bustos (1988), a fim de garantir a originalidade das informações e, dessa forma, permitir uma análise mais fidedigna da realidade das características observadas na área
Dispersão dos volumes - Sentido W-L
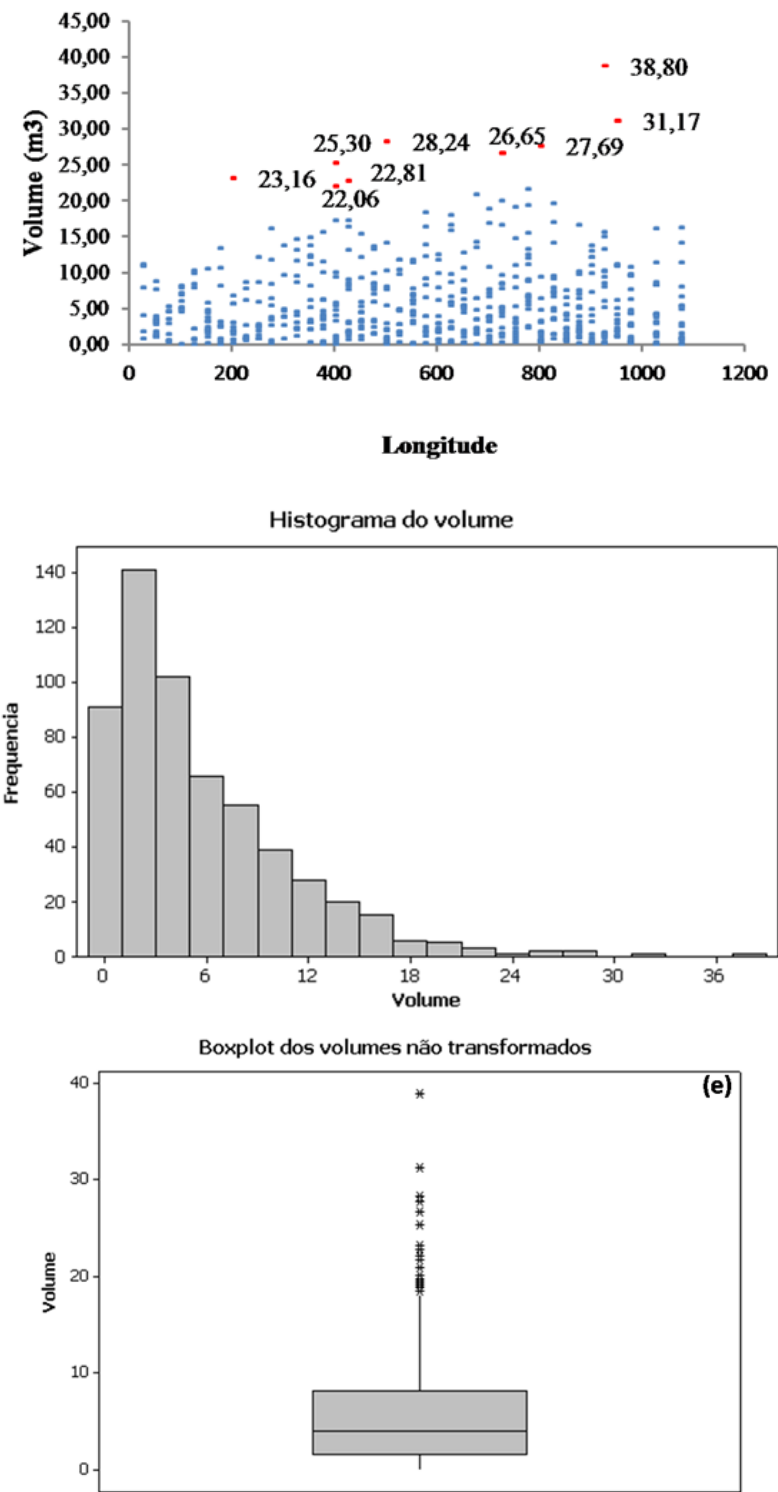

(a)
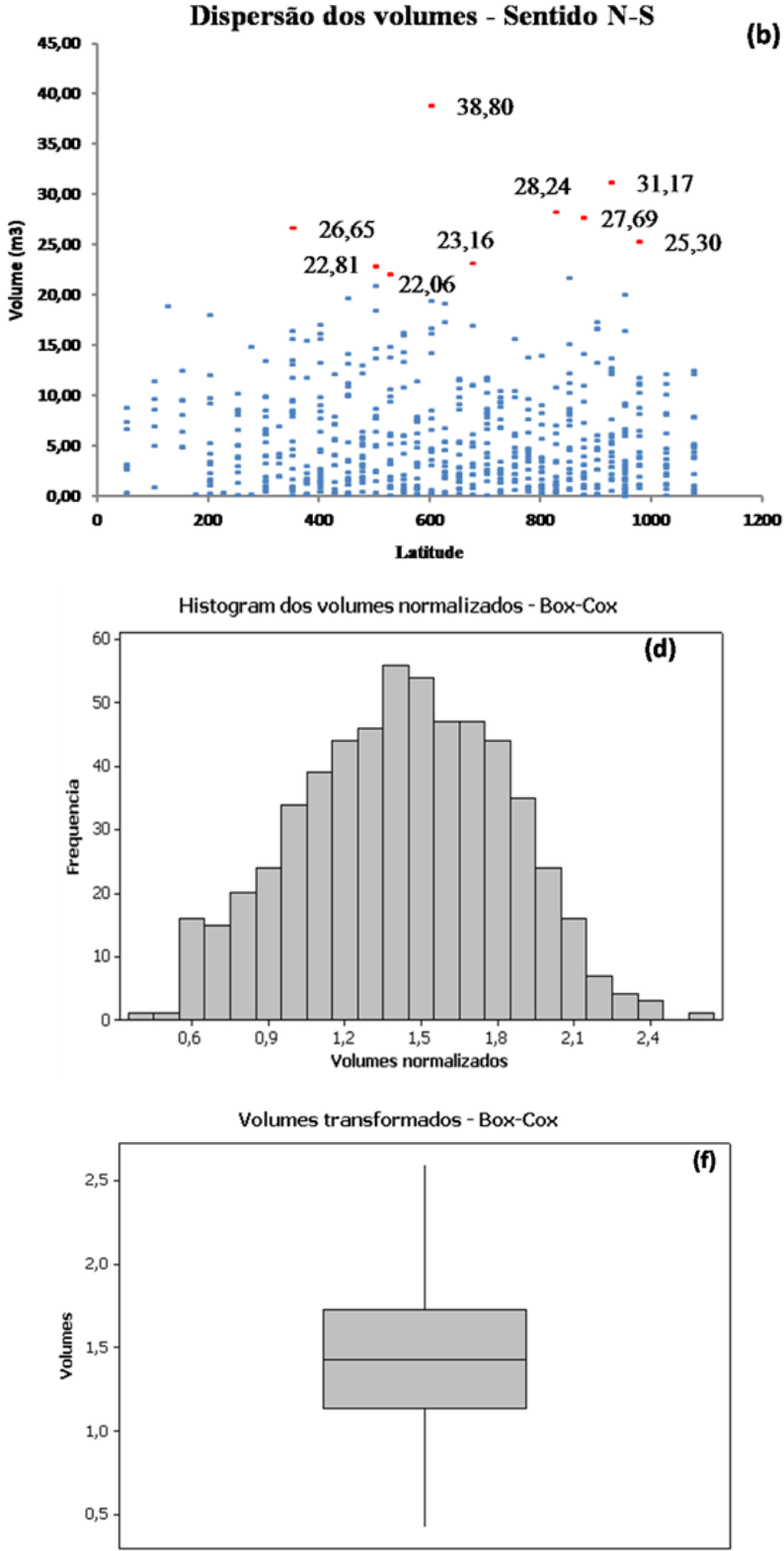

FIGURA 3 Gráficos exploratórios do volume dos resíduos, segundo os sentidos NS e WL. FIGURE 3: Graph exploratory volume of waste, according to the directions NS and WL. 
em estudo. Da mesma ideia comungam Santos et al. (2011) em estudo realizado na mesma região, destinado à definição de amostras aleatórias para a estimação do volume de espécies florestais.

Mesmo diante das evidências de subnormalidade, observadas nos Gráficos ( $\mathrm{a}, \mathrm{b}, \mathrm{c}$ e e) da Figura 3, optou-se por fazer uma confirmação através do teste de Jarque-Berra a 5\%. O resultado do teste confirmou o fato já evidenciado de que os dados não provinham de uma população com distribuição normal. Em outros termos, o teste de Jarque-Bera rejeitou a hipótese de normalidade dos dados a 5\% de significância.

O gráfico (f) da Figura 3 contém o boxplot dos dados após a transformação. Note-se que após a transformação, os dados já podem ser considerados normais.

A despeito dos valores discrepantes, os dados não apresentaram tendência com relação aos dois sentidos. Esta característica é de fundamental importância em estudos envolvendo a geoestatística, uma vez que mostra ser plausível a aplicabilidade da pressuposição intrínseca aos dados em estudo.

\section{Análise dos modelos estimados}

No ajustamento dos modelos, utilizou-se a função varioFIT do geoR, por meio dos três métodos de estimação: Mínimos Quadrados Ordinários (MQO), Mínimos Quadrados Ponderados (MQP) e

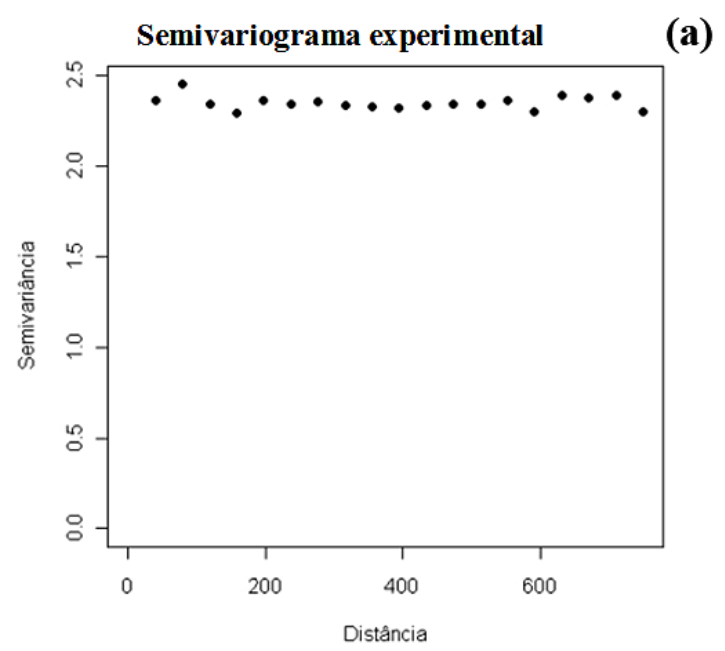

Modelo Esférico

(c)

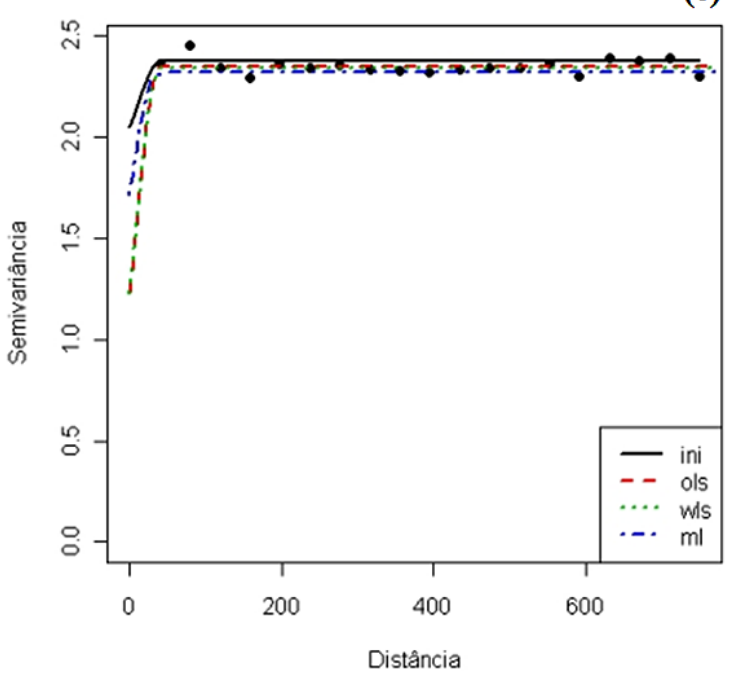

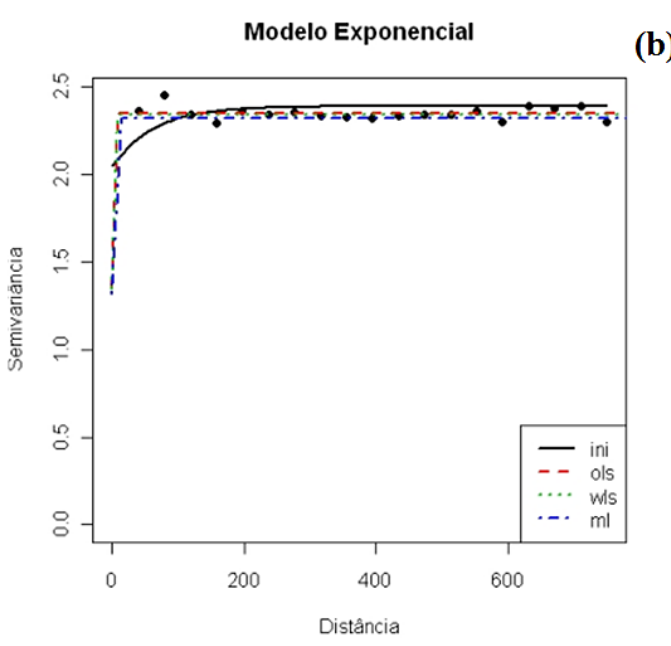

(b)

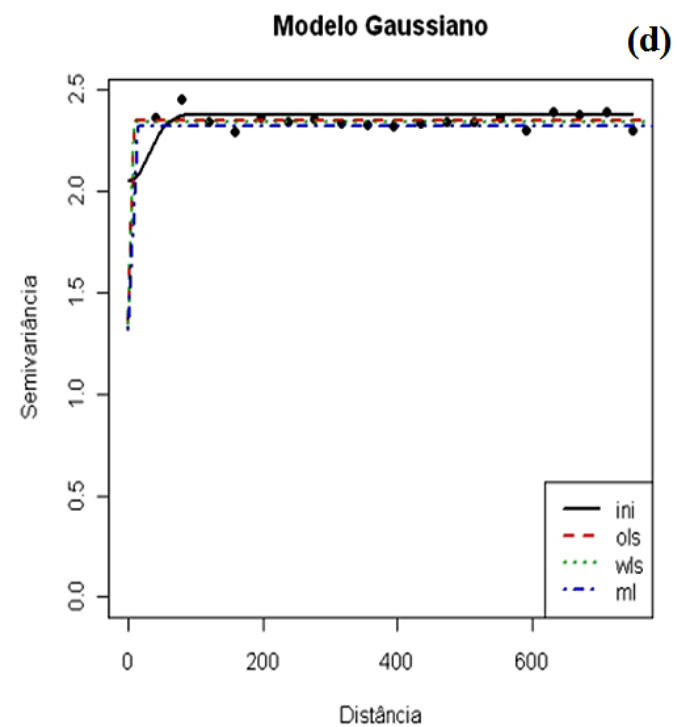

FIGURA 4 Gráficos de ajuste: (a) Semivariograma experimental; (b) Modelo exponencial; (c) Modelo Esférico e (d) Modelo Gaussiano.

FIGURE 4: Graphics adjustment: (a) Experimental semi-variogram; (b) Exponential model; (c) Spherical Model and (d) Gaussian Model. 
Máxima Verossimilhança (MV).

$\mathrm{Na}$ Figura 4 constam o semivariograma experimental, gráfico (a) e os semivariogramas ajustados, segundo os modelos teóricos, exponencial (gráfico b), esférico (gráfico c) e gaussiano (gráfico d).

Os semivariogramas apresentados neste estudo estão relacionados aos dados da área de estudo que foram transformados por Box-Cox, e que, os dados relativos aos volumes dos resíduos lenhosos tiveram suas coordenadas concentradas, sempre, nos pontos médios do quadrado formado pela malha de $50 \mathrm{~m} \times 50 \mathrm{~m}$.

Detectou-se a falta de correlação espacial entre as amostras, que é uma condição necessária para a aplicação do estimador de krigagem ordinária (KANEGAE JUNIOR, 2007). No entanto, observou-se a presença de correlação espacial para os volumes dos fustes das árvores que deram origem aos resíduos, considerada de moderada a forte, pela classificação de Cambardella et al. (1994). Acreditase, portanto, que um nível de correlação espacial dessa magnitude deve persistir também nos resíduos e, portanto, tornar factível a aplicação da KO.

Portanto, foram estimados os parâmetros dos três modelos teóricos, segundo os três métodos de ajustes: MQO, MQP e MV. Observou-se que os modelos Exponenciale Gaussiano, quando estimados pelos três métodos de ajustes, não apresentaram resultados significativos, em função da ausência de correlação espacial nas informações utilizadas no ajustamento (Figura 4, gráficos b e d), o que sugere uma forte independência das informações. Este fato, por si só, inviabiliza a utilização da KO por tais modelos, uma vez que se mostraram inadequados aos dados em estudo. Adicionalmente, observou-se que, em ambos os casos, tanto a variabilidade (sill $=\mathrm{C}_{1}$ ), quanto o alcance (a), convergem para zero (Tabela 1), evidenciando que esses modelos não se ajustaram adequadamente aos dados.

O modelo esférico (Figura 4, gráfico c) apresentou maior consistência nos ajustamentos, principalmente através do método de MQO. Neste caso, obteve-se um sill $\left(\mathrm{C}_{1}\right)$ parcial da ordem de 1,2286 , e um alcance de $\mathbf{a}=39,4738 \mathrm{~m}$. Estes resultados indicam a existência de correlação espacial, a uma distância de aproximadamente $40 \mathrm{~m}$, o que viabiliza a aplicação do método de krigagem para a distância adotada de $50 \mathrm{~m}$.

A DE, em torno de 48\%, revelou-se moderada, de acordo com o critério de Cambardella et al. (1994).

A seleção do melhor modelo foi realizada através do Critério de Informação de Akaike (AIC), como indicado nos dados da Tabela 1. Os resultados indicaram que o modelo esférico se ajustou melhor aos dados. Por isso, foi o utilizado na estimação dos pontos não amostrados.

A partir do modelo escolhido, fez-se uma estimativa para o volume nos pontos não amostrados, com base nos valores conhecidos a priori. Os resultados mostraram uma estimativa para o volume dos pontos não amostrados da ordem de 2058,43 $\mathrm{m}^{3}$, ou $21,00 \mathrm{~m}^{3} /$ ha, considerado a malha de $(50 \mathrm{~m} \mathrm{x}$ $50 \mathrm{~m}$ ). Ressalta-se que os parâmetros gerados pelo modelo, foram determinados após a transformação dos dados por Box-Cox, que foi realizada para se obter a distribuição normal dos dados. No entanto, uma estimativa feita com esses dados, antes da transformação, produziu um resultado final do volume total da área, apenas $5 \%$ superior $(6527,54$ $\mathrm{m}^{3}$ ), em relação ao volume determinado a partir da transformação dos dados pelo critério de Box-Cox $\left(6221,75 \mathrm{~m}^{3}\right)$.

Ressalta-se, todavia, que essa diferença de resultado não significa que modelos ajustados com dados transformados produzam estimativas subestimadas e vice-versa. $\mathrm{O}$ intuito foi apenas testar as duas possibilidades, uma vez que os dois procedimentos podem ser utilizados na geoestatística. Neste caso específico, o modelo aplicado aos dados não transformados gerou

TABELA1: Parâmetros estimados para cada modelo através dos métodos de ajustamento.

TABLE 1: Parameters estimated for each model using the methods of adjustment.

\begin{tabular}{ccccccccccccc}
\hline \multirow{2}{*}{ Método de ajuste } & \multicolumn{4}{c}{ Esférico } & \multicolumn{4}{c}{ Exponencial } & \multicolumn{7}{c}{ Gaussiano } \\
\cline { 2 - 13 } & $\mathrm{C}_{0}$ & $\mathrm{C}_{1}$ & $\mathrm{a}$ & $\mathrm{DE} \%$ & $\mathrm{C}_{0}$ & $\mathrm{C}_{1}$ & $\mathrm{a}$ & $\mathrm{DE} \%$ & $\mathrm{C}_{0}$ & $\mathrm{C}_{1}$ & $\mathrm{a}$ & $\mathrm{DE} \%$ \\
\hline MQO & 1,23 & 1,12 & 39,47 & 47,71 & 2,35 & 0,00 & 62,46 & 0,00 & 2,35 & 0,00 & 40,37 & 0,00 \\
MQP & 1,23 & 1,12 & 39,47 & 47,69 & 2,34 & 0,00 & 62,44 & 0,00 & 2,35 & 0,00 & 40,37 & 0,00 \\
MV & 1,72 & 0,60 & 35,36 & 26,03 & 2,32 & 0,00 & 0,00 & 0,00 & 2,32 & 0,00 & 0,00 & 0,00 \\
\hline
\end{tabular}

Fonte: Dados da pesquisa. 
TABELA 2: Síntese das estimativas dos volumes feitas através do método de Warren e Olsen e Krigagem Ordinária.

TABLE 2: Summary of estimates of the volume made by Warren and Olsen's method and Ordinary Kriging.

\begin{tabular}{cccc}
\hline Volume devido a Warren \& Olsen & Krigagem & Total & Volume por unidade de área \\
\hline \multirow{3}{*}{$4163,32 \mathrm{~m}^{3}$} & $\begin{array}{c}\text { Volume c/ dados normalizados } \\
2058,43 \mathrm{~m}^{3}\end{array}$ & $6221,75 \mathrm{~m}^{3}$ & $71,085 \mathrm{~m}^{3} / \mathrm{ha}$ \\
\cline { 2 - 4 } & $\begin{array}{c}\text { Volume c/ dados não normalizados } \\
2364,22 \mathrm{~m}^{3}\end{array}$ & $6527,54 \mathrm{~m}^{3}$ & $74,58 \mathrm{~m}^{3} / \mathrm{ha}$ \\
\hline
\end{tabular}

Fonte: Dados da pesquisa.

estimativas mais próximas do valor real.

Com efeito, a estimação do volume total da área em estudo pode ser vista sob dois aspectos. No primeiro, considera-se o resultado gerado pelo método proposto de Warren e Olsen, acrescido dos valores obtidos através da $\mathrm{KO}$, a partir dos dados transformados. No segundo, consideraramse os valores dos volumes obtidos através das linhas interceptadoras, acrescidos dos valores obtidos pela $\mathrm{KO}$, considerando os valores brutos (não transformados). A Tabela 2 contém a síntese dos resultados obtidos neste estudo, mostrando as estimativas geradas pelos dois métodos.

Finalmente, o valor obtido para o volume total do povoamento florestal em estudo, aproximou-se do volume de resíduo efetivamente retirado da área pela empresa exploradora, da ordem de $6867,01 \mathrm{~m}^{3}$, ou seja, a discrepância observada foi de apenas $339,47 \mathrm{~m}^{3}(5,2 \%)$ entre o valor estimado e o valor real.

\section{CONCLUSÕES}

Os dados obtidos da exploração de floresta nativa atenderam aos pressupostos de normalidade e de correlação espacial, respectivamente, da Estatística Clássica e da Geoestatística. Concluiuse que, mesmo com os dados apresentado um nível de correlação espacial mediano, as estimativas do volume final obtidas com dados normalizados e não normalizados apresentaram bons resultados.

As estimativas geradas a partir dos dados não normalizados apresentaram resultados significativamente mais próximos dos valores reais dos resíduos. Contudo, tal desempenho pode não se repetir em outras aplicações.

A aplicação conjunta dos métodos de Warren e Olsen e da Krigagem Ordinária, mostrouse adequada, uma vez que gerou uma estimativa satisfatória para os volumes de resíduos nos pontos não amostrados, a partir dos dados amostrados.

Finalmente, conclui-se que entre os modelos de ajustamento aplicados ao semivariograma experimental, o modelo esférico apresentou o melhor resultado, independentemente do método de estimação utilizado.

\section{REFERÊNCIAS BIBLIOGRÁFICAS}

BUSTOS, O. H. Outliers e robustez. Revista Brasileira de Estatística, v. 49, n. 192, p. 1-168, 1988.

CAMBARDELLA, C. A. et al. Field scale variability of soil properties incentral Iowa soils. Soil Science Society of America Journal, v. 58, n. 5, p. 15011511, 1994.

DAVIS, E.; IERAPETRITON, M. A Kriging Method for the Solution of Nonlinear Programs with Black-Box Functions. AIChE Journal, v. 53, n. 8, p. 2001-2012, 2007.

DIGGLE, P. J.; RIBEIRO JÚNIOR, P. J. Modelbased geostatistics. In: SIMPÓSIO NACIONAL DE PROBABILIDADE E ESTATÍSTICA, 14., 2000, Caxambú. Resumos... Caxambú: Associação Brasileira de Estatística, 2000, p. 192.

GREENE, W. H. Econometrics analisys. New York: Macmillan Publishing Company, 1993.

IMAI, N. N. et al. Análise comparativa da interpolação por krigagem Ordinária e krigagem por indicação no caso de ervas daninhas em cultura de soja. Universidade Estadual Paulista - Unesp. Faculdade de Ciências e Tecnologia Departamento de Cartografia, 2003. Disponível do site: WWW.cartografia.org.br. Acesso: 19/05/2009. ISAAKS, E. H; SRIVASTAVA, R. M. An introduction to applied geostatistics. New York: Oxford Univ. Press, 1989.

JOURnEL, A. G.; HUIJBREGTS, C. J. Mining 
geostatistics. London: Academic Press, 1978.

KANEGAE JUNIOR, H. et al. Avaliação da continuidade espacial de características dendrométricas em diferentes idades de povoamentos clonais de Eucalyptus sp.. Revista Árvore, v. 31, n. 5, p. 859-866, 2007.

LANDIM, P. M. M. Análise estatística de dados geológicos - São Paulo: Fundação Editora da UNESP, 1998.

MELLO, J. M. Geoestatística aplicada ao inventário florestal. Tese (Doutorado em Estatística) - Escola Superior Luiz de Queiroz, Piracicaba, 2004.

PARÁ. Secretaria de Estado de Planejamento, Orçamento e Finanças do Estado do Pará. Portal Amazônia. Disponível em: <http://portalamazonia. globo.com/artigos>. Acesso em: 05 maio 2009.

QUEIROZ, J. C. B. Uso da geoestatística para quantificar o risco de contaminação por metais pesados no porto de Santana-Amapá. Tese (Doutorado em Estatística) - Universidade Estadual Paulista, Rio Claro, 2003.

SANTANA, A. C. de. Métodos quantitativos em economia: elementos e aplicações. Belém: UFRA, 2003.

SANTOS, C. L. A. et al. Mapeamento de recifes submersos na costa do Rio Grande do Norte, NE Brasil: Macau a Maracajau. Revista Brasileira de Geografia, v. 25, n. 1, p. 27-36, 2007.

SANTOS, P. C. et al. O emprego da geoestatística na determinacão do tamanho "ótimo" de amostras aleatórias com vistas à obtenção de estimativas dos volumes dos fustes de espécies florestais em Paragominas, estado do Pará. Acta Amazônica, v. 41, n. 2, p. 213-222, 2011.

VAN WAGNER, C. E. V. The line intersect method in forest fuel sampling. Forest Science, v. 14, n. 1, p. 20-26, 1968.

WANG, Q; NI, J.; TENHUNEN, J. Application of a geographically-weighted regression analysis to estimate net primary production of Chinese forest ecosystems. Glob Ecol Biogeogr, v. 14, n. 4, p. 379-393, 2005.

WARREN, W. G.; OLSEN, P. F. A line intersect technique for assessing logging waste. Forest Science, v. 10, n. 3, p. 267-276, 1964. 